\title{
ON A METHOD OF DEALING WITH THE INTERSECTIONS OF PLANE CURVES*
}

BY

\author{
F. S. MACAULAY
}

\section{Introduction.}

1. The following is an investigation of certain theorems which $I$ gave in the Proceedings of the London Mathematical Society, vol. 31 (1899), pp. 381-423, and which were later discussed in a simpler manner by Dr. C. A. Scotr in the Transactions of the American Mathematical Society, vol. 3 (1902), pp. 216-263. Initials, $P$ and $T$, are used below for reference to these two sources respectively.

2. Noether's fundamental theorem states that certain conditions are sufficient in order that a polynomial $F$ in two variables $x, y$ may be of the form $A f+B \phi$, where $f, \phi$ are given polynomials, and $A, B$ undetermined polynomials. These conditions may be thus expressed: Change the origin to any point $a, b$, i. e., substitute $x+a, y+b$ for $x, y$ in $F, f, \phi$, and expand in ascending powers of $x, y$; then, whatever finite values $a, b$ may have, $F$ must be of the form $A^{\prime} f+B^{\prime} \phi$, where $A^{\prime}, B^{\prime}$ are undetermined infinite power series, which are entirely different for different origins. Any origin at a point of intersection of the curves $f, \phi$ supplies a certain number of conditional equations for the coefficients of $F$; the conditions which different origins supply are quite independent of one another, and there is no limit to the number of conditions which a single origin may supply. On the other hand, any origin which is not a point of intersection of $f, \phi$ supplies no conditions; for if $f$ (say) does not vanish at the origin, $F / f$ is a power series, so that $F$ is of the form $A^{\prime} f$, and therefore of the form $A^{\prime} f+B^{\prime} \phi$.

The two memoirs mentioned in $\S 1$ relate to the conditions which are supplied for $F$ in the most general case when the origin is a point at which the curves $f, \phi$ have multiple points of any order and complexity, and contact of any kind.

3. We modify the notation by writing $S, C_{1}, C_{2}$ for $F, f, \phi\left(C_{1}, C_{2}\right.$ being either given polynomials or power series), and $P_{1}, P_{2}$ for $A^{\prime}, B^{\prime}$. We also write

$$
C_{1}=\Sigma a_{q}^{p} x^{p-q} y^{q}, \quad C_{2}=\Sigma b_{q}^{p} x^{p-q} y^{q}, \quad S=\Sigma z_{q}^{p} x^{p-q} y^{q} \quad(p \geqq q \geqq 0) .
$$

* Presented to the Society at the St. Louis meeting, September 16-17, 1904. Received for publication November 13, 1903. 
The subject of investigation is therefore the whole system of identical equations which must be satisfied by the coefficients $z_{q}^{p}$ of $S$ in order that the identity $S=C_{1} P_{1}+C_{2} P_{2}$ may exist, where $P_{1}, P_{2}$ are undetermined power series.

It should be observed first of all that if the equation $\Sigma \alpha_{q}^{p} z_{q}^{p}=0$ is identically satisfied, then all the equations $\Sigma \alpha_{q}^{p} z_{q-m}^{p-l}=0(l \geqq m \geqq 0)$ are also satisfied ; for

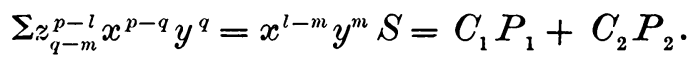

It is easily proved conversely that the equation $\Sigma \alpha_{q}^{p} z_{q}^{p}=0$, and consequently the one-set system consisting of the prime equation $\Sigma \alpha_{q}^{p} z_{q}^{p}=0$ and all its derivates $* \sum \alpha_{q}^{p} z_{q-m}^{p-l}=0$, is identically satisfied, provided the same one-set system is satisfied for $C_{1}$ and $C_{2}$. Thus the whole system of identical equations satisfied by the z's consists of all the one-set systems which are satisfied by the coefficients of $C_{1}$ and of $C_{2}$; and we have simply to investigate the properties of the one-set systems of equations which are satisfied by both $C_{1}$ and $C_{2}$.

4. The work given below covers practically the same ground as Miss Scotr's memoir $T$; and aims at dealing with the subject in an exact and general manner, although in many places the argument is based on particular examples. I have made several attempts at different times to place the theory on a purely algebraic foundation (cf. $P \S 8$ ); but should probably not have succeeded except for the help derived from $T$. I have followed $T$ in regarding the oneset theorem as the fundamental one, from which the whole subject is most naturally developed, and have found the diagrams of $T \S 32$ of the greatest assistance in proving more than one important property ( $\S 15-19)$. The principal theorems proved are:

(i) The one-set theorem ( $\S 7-13)$. All the one-sets which two given curves, or series of coefficients, satisfy make up a single one-set; provided the two curves have not a common branch through the origin $(\S 20)$.

Conversely, the complete solution of any one-set system of equations is expressible by means of two particular solutions $(\S 14)$. This converse is not proved in $T$, and is not a consequence of the theorem itself.

(ii) The t-set theorem ( $\S 22,23)$. All the one-sets which $t+1$ independent curves, or series, satisfy make up $t$ independent one-sets, i. e., a $t$-set; provided une $t+1$ curves have not all a common branch through the origin. This may be said to include its own converse.

(iii) The number of (ordinary) points to which the whole intersection of any two given curves at the origin is equivalent is equal to the number of independent equations in the one-set determined by the two curves $(\S 18)$.

In the proof of (i) in $T \S \S 14-24$, so many different cases are encountered as

\footnotetext{
* $\Sigma \alpha_{q}^{p} z_{q}^{p-1}, \Sigma \alpha_{q}^{p} z_{q}^{p-2}, \cdots$ are called the 1 st, $2 \mathrm{~d}, \cdots x$-derivates of the expression $\Sigma \alpha_{q}^{p} z_{q}^{p}$, and $\Sigma \alpha_{q}^{p} z_{q-1}^{p-1}, \Sigma \alpha_{q}^{p} z_{q-2}^{p-2}, \cdots$, the 1 st, $2 d, \cdots y$-derivates. Also $E$ (or $\left.E_{0}^{0}\right)$ and $E_{m}^{l}$ are written respectively for $\Sigma_{\alpha_{q}^{p}}^{p} z_{q}^{p}$ and $\Sigma_{\alpha_{q}^{p}} z_{\psi-m}^{p-l}$.
} 
to create a doubt whether some possibilities may not have been overlooked, such as, for example, the possibility of $A_{0}$ vanishing in $T \S 20$. I believe, however, that no important point is omitted; although I am not satisfied that all the points noted are sufficiently proved. In regard to (ii), I think the proof given in $T \S 25$ is quite incorrect, and that (ii) cannot be proved apart from difficult. considerations which are not involved in (i). An error also occurs in the proof of (iii) in $T \S 27$, which invalidates the proof of $T \S 29$.

5. The proof of (ii) in $T \S 25$ is based on the assumption that if $m+1$ one-sets of degree $p$ are satisfied by $t+1$ given independent curves, then the process of ascent for two of the curves will yield $m$ equations of degree $p+1$ (the memoir says $p$ which I take to be a misprint for $p+1$ ). But the process of ascent, as I understand it, is a general process, applicable to all curves which satisfy the $m+1$ one-sets of degree $p$; and it is only the result that is applied to particular curves. This general process, when applied to $m+1$ one-sets of degree $p$, does not in general give $m$ equations of degree $p+1$. What must be shown is that if all the one-sets of degree $\leqq p$ satisfied by the curves make up an $(m+1)$ set, where $m+1>t$, then the process of ascent yields one or more equations of degree $p+1$, containing sufficient undetermined constants to allow of their being satisfied by the $t+1$ curves. If the above criticism is due to a misapprehension of the meaning of $T \S 25$, it may still be maintained that the reasoning falls far short of a complete proof of theorem (ii).

6. The statement of $T \S 27$ is: "If an expression $E$ and its first $k y$-derivates $E_{1}^{1}, E_{2}^{2}, \ldots, E_{k}^{k}$, as well as all the $x$-derivates of these, vanish for two curves that have at the origin a multiple point of order $k$, then all the derivates of $E$ vanish, so that $E$ is a member of the one-set system proper to the point." If true, this would materially simplify the proof of (iii) in $P \S \S 12-17$; but it is assumed that no equations which involve the coefficients of powers of $y$ only can be satisfied for both curves, when the axes have no special relation to the curves. This is clearly a mistake, when, as is the case in $T \S 27$, the equations do not belong to a one-set system.

Take, as an example, the point whose prime equation is

$$
z_{0}^{3}+m z_{1}^{3}+m^{2} z_{2}^{3}+m^{3} z_{3}^{3}=0,
$$

the derivates of which are

$$
z_{0}^{2}+m z_{1}^{2}+m^{2} z_{2}^{2}=z_{0}^{1}+m z_{1}^{1}=z_{0}^{0}=0 .
$$

The two curves $C_{1}, C_{2}$ which determine this point have only a linear branch through the origin, so that $k=1$. Now take

then

$$
E=z_{0}^{4}+m z_{1}^{4}+m^{2} z_{2}^{4}+m^{3} z_{3}^{4}+m^{4} z_{4}^{4}+\alpha z_{4}^{4}+\beta z_{3}^{3}+\gamma z_{2}^{2}+\delta z_{1}^{1} ;
$$

$$
E_{1}^{1}=n \iota z_{0}^{3}+m^{2} z_{1}^{3}+m^{3} z_{2}^{3}+m^{4} z_{3}^{3}+\alpha z_{3}^{3}+\beta z_{2}^{2}+\gamma z_{1}^{1} .
$$


All the $x$-derivates of $E$ and $E_{1}^{1}$ vanish for $C_{1}$ and $C_{2}$, and $\alpha, \beta, \gamma, \delta$ can be so chosen that $E$ and $E_{1}^{1}$ also vanish for $C_{1}$ and $C_{2}$; yet $E$ is not a member of the one-set system proper to the point. It may be noticed that

$$
E_{1}^{1}-m E_{0}^{1}=\alpha z_{3}^{3}+\beta z_{2}^{2}+\gamma z_{1}^{1}
$$

but this expression can, and does, vanish for both curves, notwithstanding the fact that it involves coefficients of powers of $y$ only.

\section{The one-set theorem.}

7. Let $C_{1}=\Sigma a_{q}^{p} x^{p-q} y^{q}, C_{2}=\Sigma b_{q}^{p} x^{p-q} y^{q}$, be two given polynomials or power series in which no terms are present of degree less than $i$. We shall be concerned chiefly with the series of elements formed by their coefficients, viz.:

$$
\begin{gathered}
a_{0}^{i}, a_{1}^{i}, \ldots, a_{i}^{i}, a_{0}^{i+1}, a_{1}^{i+1}, \ldots, a_{i+1}^{i+1}, a_{0}^{i+2}, \ldots, \\
b_{0}^{i}, b_{1}^{i}, \ldots, b_{i}^{i}, b_{0}^{i+1}, b_{1}^{i+1}, \ldots, b_{i+1}^{i+1}, b_{0}^{i+2}, \ldots
\end{gathered}
$$

In each series the first $\frac{1}{2} i(i+1)$ elements vanish, and also all elements outside a certain range if $C_{1}, C_{2}$ are polynomials. All these vanishing elements should, however, be considered as belonging to the series. We may assume that no other elements vanish.* It is only essential, however, that.we assume $a_{i}^{i}$ not to vanish.

We enquire first, what are the one-sets which both series satisfy? In order that $\Sigma \zeta_{q}^{p} z_{q}^{p}=0$ may be one it is necessary and sufficient $(\S 3)$ that the equation $\Sigma \zeta_{q}^{p} z_{q}^{p}=0$, and all its derivates, should be satisfied both when $z_{q}^{p}=a_{q}^{p}$, and when $z_{q}^{p}=b_{q}^{p}$. This gives at once the equations for the $\zeta$ 's; and the general solution (involving arbitrary linear parameters), being substituted in $\Sigma \zeta_{q}^{p} z_{q}^{p}=0$, will give all the equations (both prime and derivate) of the required one-set systems, by equating to zero the coefficients of the arbitrary parameters involved in the $\zeta$ 's. Moreover any particular solution of the $\zeta$ 's supplies the coefficients of a one-set $\Sigma \zeta_{p}^{p} z_{q}^{p}=0$, which may however be a derivate of a more extensive one-set.

8. The $\zeta$ equations are

$$
\Sigma \zeta_{q}^{p} a_{q}^{p}=0, \quad \Sigma \zeta_{q}^{p} a_{q}^{p-1}=0, \quad \Sigma \zeta_{q}^{p} a_{q-1}^{p-1}=0, \quad \Sigma \zeta_{q}^{p} a_{q}^{p-2}=0, \cdots,
$$

and

$$
\Sigma \zeta_{q}^{p} b_{q}^{p}=0, \quad \Sigma \zeta_{q}^{p} b_{q}^{p-1}=0, \quad \Sigma \zeta_{q}^{p} b_{q-1}^{p-1}=0, \quad \Sigma \zeta_{q}^{p} b_{q}^{p-2}=0, \quad \cdots,
$$

which may also be written

$$
\begin{aligned}
& \Sigma a_{q}^{p} \zeta_{q}^{p}=0, \quad \Sigma a_{q}^{p} \zeta_{q}^{p+1}=0, \quad \Sigma a_{q}^{p} \zeta_{q+1}^{p+1}=0, \quad \Sigma a_{q}^{p} \zeta_{q}^{p+2}=0, \cdots, \\
& \Sigma b_{q}^{p} \zeta_{q}^{p}=0, \quad \Sigma b_{q}^{p} \zeta_{q}^{p+1}=0, \quad \Sigma b_{q}^{p} \zeta_{q+1}^{p+1}=0, \quad \Sigma b_{q}^{p} \zeta_{q}^{p+2}=0, \quad \cdots
\end{aligned}
$$

* If $C_{1}, C_{2}$ are transformed to $C_{1}^{\prime}, C_{2}^{\prime}$ when $x=l x^{\prime}+m y^{\prime}, y=l^{\prime} x^{\prime}+m^{\prime} y^{\prime}\left(l / m \neq l^{\prime} / m^{\prime}\right)$, it can be easily proved that a one-to-one correspondence exists between the one-sets satisfied by $C_{1}, C_{2}$ and the one-sets satisfied by $C_{1}^{\prime}, C_{2}^{\prime}$. The same is true for the transformation $x=l x^{\prime}+m y^{\prime}+$ higher terms, $y=l^{\prime} x^{\prime}+m^{\prime} y^{\prime}+$ higher terms. 
These are best exhibited by simply writing down the array of the coefficients :

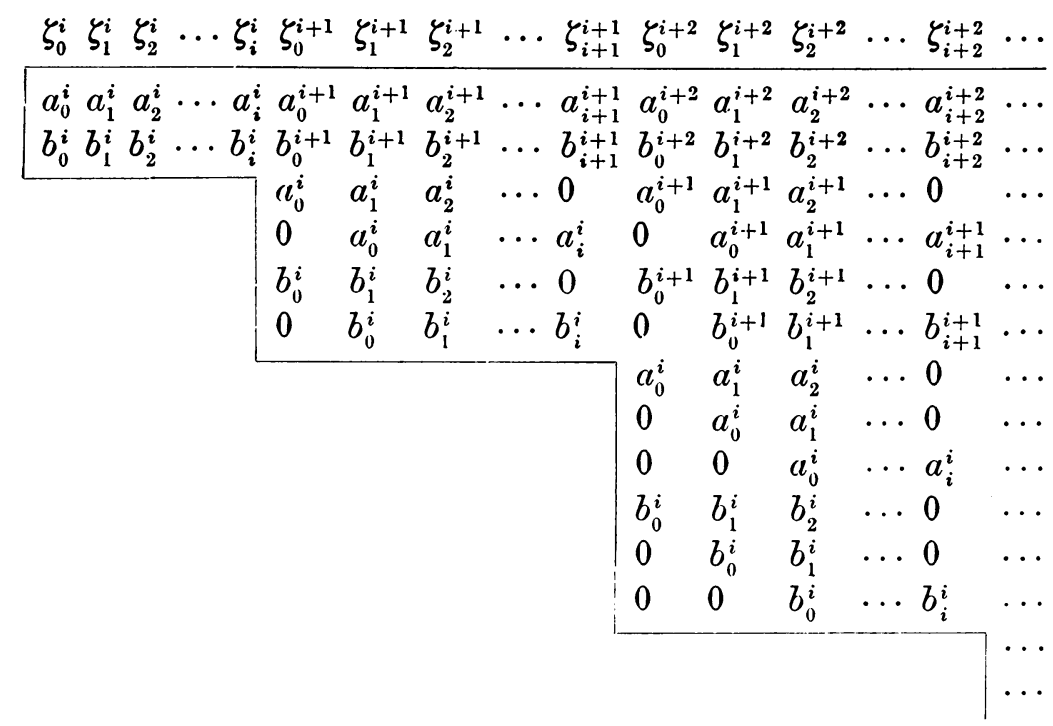

Thus the equations divide themselves into sets, according to the index of $\zeta$ in the leading terms. Corresponding to these we have sets of elements, and leading elements of a set, viz. the array of coefficients of $\zeta$ 's with lowest index in the set. This index is called the degree of the set and of the corresponding set of equations. As they stand, the sets of elements contain two, four, six, etc., rows respectively; but we may have to modify and diminish the sets, while keeping the whole array or system of equations complete, in order that, in the final form, the rows of leading elements in each set may be linearly independent. We will suppose this done for the present, and proceed to mention the chief properties of the equations, leaving the proofs of (ii), (iv), and (vi) to be given later.

(i) Each $\zeta$ with an index less than $i$ is arbitary, since its coefficient is zero in all the equations.

Each $\zeta$ with an index greater than the limit index is zero, as explained in (iii).

(ii) Each set in the modified array contains either one or two more rows (or equations) than the preceding set ( $\$ 9)$. If only one more, the set is said to be irregular; if two more, the set is regular.

The first set will be irregular if $C_{1}, C_{2}$ have precisely the same tangents at the origin. If the first $j-i$ sets are irregular, an integral polynomial $S$ exists such that $C_{2}+C_{1} S$ has a multiple point of order $j$ at the origin. Also, as $C_{2}$ may be replaced by $C_{2}+C_{1} S$ for the purpose of finding the one-sets of $C_{1}, C_{2}$, this case corresponds to two curves having multiple points of different orders $i, j$ at the origin. 
(iii) There cannot be more than $i$ regular sets altogether; and at the $i$ th regular set the $\zeta$ 's vanish. For the number of $\zeta$ 's in the leading terms of the $i$ th regular set of equations is equal to the number of equations in that set; and the same is true for every later set, by (ii). Hence all $\zeta$ 's with an index greater than a certain limit index (viz., the index of the $\zeta$ 's in the leading terms of the set preceding the $i$ th regular set) are expressible in terms of $\zeta$ 's whose indices exceed any assigned index, however great, and so cannot be retained. Thus all $\zeta$ 's which occur in the $i$ th regular set must be taken to be zero.

This may also be proved in another way. Let $n$ be the index of the $\zeta$ 's in the leading terms of the $i$ th regular set, so that $n+1$ is the number of rows of the set. Now the value of $z_{q}^{p}$ (the coefficient of $x^{p-q} y^{q}$ in $C_{1} P_{1}+C_{2} P_{2}$, $P_{1}, P_{2}$ being arbitrary) is obtained by taking the sum of the products of a column of arbitrary elements (or parameters) with the column of elements corresponding to $\zeta_{q}^{p}$. Hence, in $z_{0}^{n}, z_{1}^{n}, \ldots, z_{n}^{n}, n+1$ significant parameters are involved, viz., the elements of the arbitrary column corresponding to the $n+1$ rows of the ith regular set, which do not appear at all in the $z$ 's with index $<n$. Thus $z_{0}^{n}, z_{1}^{n}, \cdots, z_{n}^{n}$ cannot appear permanently in any identical equation (after their coefficients have been collected), since the $n+1$ significant parameters cannot be eliminated; and, for the same reason, no $z$ with an index $\geqq n$ can appear. But $\Sigma \zeta_{q}^{p} z_{q}^{p}=0$ is an identical equation; hence $\zeta_{q}^{p}$ vanishes when $p \geqq n$.

(iv) If every set after the $k$ th regular set is irregular, where $k<i$, then $C_{1}$, $C_{2}$ are divisible by a common power series $K$ having a multiple point of order $i-k$ at the origin ( $(20)$. In this case the limit index mentioned in (iii) does not exist; and the whole intersection of $C_{1}, C_{2}$ at the origin is made up of $K$ and the finite intersection of $C_{1} / K$ and $C_{2} / K$ at the origin.

(v) If $\zeta_{q}^{p}=\alpha_{q}^{p}$ is one solution of the $\zeta$ equations, it is easily seen that $\zeta_{q}^{p}=\alpha_{q}^{p+1}, \zeta_{y}^{p}=\alpha_{q+1}^{p+1}, \ldots \zeta_{q}^{p}=\alpha_{q+m}^{p+l}(l \geqq m), \cdots$ are other solutions $(\S 8$, line 6$)$.

(vi) If $\zeta_{q}^{p}=\alpha_{q}^{p}$ is any particular solution such that the $\alpha$ 's with the limit index do not vanish, then the several solutions $\zeta_{q}^{p}=\alpha_{q+s}^{p+r}(r \geqq s)$ give the complete solution, i. e., the general solution is expressible by means of a single solution, viz., $\zeta_{q}^{p}=\Sigma \lambda_{s}^{r} \alpha_{q+s}^{p+r}$, where the $\lambda$ 's are arbitrary ( $\$ 10-13$ ).

This last is the fundamental property of the $\zeta$ equations; and from it follows the one-set theorem $\S 4$ (i). For all the one-sets which the series $a, b$ satisfy are included in $\Sigma \zeta_{q}^{p} z_{q}^{p}=0$, when $\zeta_{q}^{p}$ is given its general value; but

$$
\Sigma \zeta_{q}^{p} z_{q}^{p}=\Sigma z_{q}^{p} \Sigma \lambda_{s}^{r} \alpha_{q+s}^{p+r}=\Sigma \lambda_{s}^{r} \Sigma \alpha_{q+s}^{p+r} z_{q}^{p}=\Sigma \lambda_{s}^{r} \Sigma \alpha_{q}^{p} z_{q-s}^{p-r}
$$

so that the general equation $\Sigma \zeta_{q}^{p} z_{q}^{p}=0$ gives the one-set $\Sigma \alpha_{q}^{p} z_{q}^{p}=0$ and its derivates, and nothing more. This reasoning is not affected by the fact that the number of the $\lambda$ 's is not the least number of arbitrary parameters by means of which the general solution for $\zeta$ can be expressed.

9. We have now to examine the result of modifying the array of the coeffi- 
cients $(\S 8)$ so as to make the rows of leading elements in each set linearly independent. Suppose the first $p$ sets are regular, and the $\overline{p+1}$ th irregular. The leading elements of the $p$ th and $\overline{p+1}$ th sets are

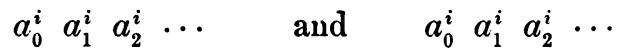

$$
\begin{aligned}
& \begin{array}{llllllllllll}
0 & a_{0}^{i} & a_{1}^{i} & \ldots & 0 & a_{0}^{i} & a_{1}^{i} & \ldots
\end{array} \\
& p \text { rows } \quad p+1 \text { rows } \\
& \begin{array}{llllllllllllll}
b_{0}^{i} & b_{1}^{i} & b_{2}^{i} & \ldots & b_{0}^{i} & b_{1}^{i} & b_{2}^{i} & \cdots
\end{array} \\
& \begin{array}{lllllllllll}
0 & b_{0}^{i} & b_{1}^{i} & \cdots & 0 & b_{0}^{i} & b_{1}^{i} & \cdots
\end{array} \\
& p \text { rows, } \quad p+1 \text { rows; }
\end{aligned}
$$

and in the former the rows are linearly independent, but not in the latter. The latter array differs from the former only by the addition of the last $a$ row, the last $b$ row, and the last column. Hence the first $p$ rows $a$ and first $p$ rows $b$ in in the latter array are independent. These supply none but zero elements for the last column; hence the last $a$ row, which has a non-vanishing element $a_{i}^{i}$ in the last column, is an additional independent row. The last row of all must then be dependent on the first $2 p+1$ rows, which are independent. We can therefore multiply the first $2 p+1$ rows by such quantities that, when added to the last row, the leading elements in that row all vanish. The row of the whole array becomes then a row belonging to the $\overline{p+2}$ th set, with leading terms $\zeta^{i+p+1}$, and is

$$
c_{0}^{i+p+1}, c_{1}^{i+p+1}, \ldots, c_{i+p+1}^{i+p+1}, c_{0}^{i+p+2}, \ldots, c_{i+p+2}^{i+p+2}, \cdots
$$

The leading elements of the $\overline{p+2}$ th set consist now of $p+2$ rows $a_{0}^{i}, \ldots, a_{i}^{i}, p+2$ rows $b_{0}^{i}, \ldots, b_{i}^{i}$, and 1 row $c_{0}^{i+p+1}, \ldots, c_{i+p+1}^{i+p+1}$. By treating the first $p+1$ rows $a$ and first $p+1$ rows $b$ in the same way as in the $p+1$ th set, the $p+1$ th row $b$ is replaced by a row $c$ in the $\overline{p+3}$ th set; similarly by means of the $p+1$ rows $a$ after the first, and the $p+1$ rows $b$ after the first, the

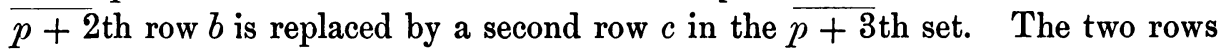
$c$ in the $\overline{p+3}$ th set are

$$
\begin{array}{lllll}
c_{0}^{i+p+1} & c_{1}^{i+p+1} \cdots c_{i+p+1}^{i+p+1} & 0 & c_{0}^{i-p+2} & c_{1}^{i+p+2} \cdots \\
0 & c_{0}^{i+p+1} \cdots c_{i+p}^{i+p+1} & c_{i+p+1}^{i+p+1} & 0 & c_{0}^{i+p+2} \cdots
\end{array}
$$

Thus the leading elements in the $\overline{p+2}$ th set reduce to $p+2$ rows $a, p$ rows $b$, and 1 row $c$. Those of the $\overline{p+3}$ th set similarly reduce to $p+3$ rows $a, p$ rows $b$, and 2 rows $c$; and so on for the remaining sets, the number of $a$ rows and $c$ rows increasing by 1 in each set, and the number of $b$ rows remaining stationary and equal to $p$. Thus the effect of an irregularity in the $\overline{p+1}$ th set 
is to reduce the whole number of rows in each set from the $\overline{p+1}$ th onwards by 1 , the $q$ th set $(q>p)$ having its $q$ rows $b$ altered to $p$ rows $b$ and $q-p-1$ rows $c$.

We may now consider the effect of a second irregularity, which may occur at

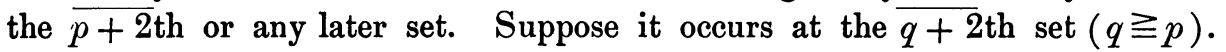
As before, the last row of leading elements of the $q+2$ th set, i. e., the $\overline{q-p+1}$ th row $c$, must be dependent on the rest, and the row of the whole array is replaced by a row $d$ belonging to the $\overline{q+3}$ th set. The same reasoning applies as before, the only difficulty occurring with the $b$ rows, the number of which remains stationary after the $p$ th set. We may, however, reinsert the $b$ rows which have disappeared for the purpose of deducing the $d$ rows. The result is that the $q+3$ th set will have $q+3$ rows $a, p$ rows $b, q-p$ rows $c$, and 1 row $d$; and in succeeding sets the number of $a$ and $d$ rows will increase by one in each set, and the number of $b$ and $c$ rows will remain stationary, while the whole number of rows in each set from the $q+2$ th onwards will be again diminished by 1 . This process of modification must be continued until we arrive at the $i$ th regular set with leading elements linearly independent. From and after this point the $\zeta$ 's vanish $(\S 8$, iii). The system of $\zeta$ equations may then be said to be prepared for solution. The solution is obtained by starting with the set preceding the $i$ th regular set, and working backwards through the sets.

10. We have already seen in $\S 8(\mathrm{v})$ that a single solution $\zeta_{q}^{p}=\alpha_{q}^{p}$ gives other solutions, viz., the $(s+1)$ th solution of the $(r+1)$ th set of solutions is $\zeta_{q}^{p}=\alpha_{q+s}^{p+r}(r \geqq s)$. The array of these solutions, supposing $l$ the limit index, is

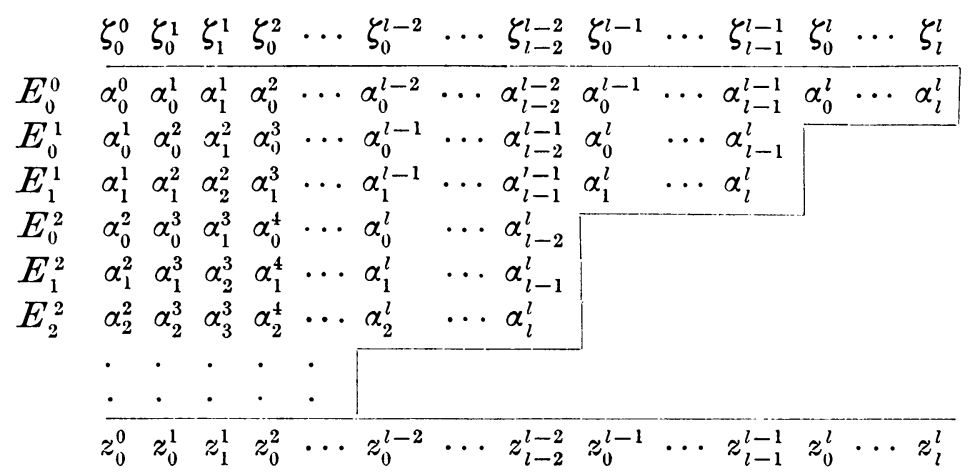

This is also the array of the coefficients of the one-set system of equations consisting of $E_{0}^{0}=\Sigma \alpha_{q}^{p} z_{q}^{p}=0$ and all its derivates $E_{s}^{r}=0$, which we will call the $z$ equations. The $z$ equations divide into sets, according to the degree, or index of $z$ in the leading terms, giving this name to the terms with highest index in the $z$ equations, and to the terms with lowest index in the $\zeta$ equations. We modify the $z$ array in the same way as the $\zeta$ array, so that the rows of leading elements in each set may be linearly independent. The reasoning of $\$ 9$ shows that, after modifica- 
tion, the number of rows in any set is at most 1 more than in the previous set; if it is 1 more, the set is called regular, otherwise it is irregular. An irregulur set has the same number of rows as the preceding set. For, in the set of equations of degree $n$, we cannot eliminate all the leading terms $z_{0}^{n}, z_{1}^{n}, \cdots z_{n}^{n}$, since the rows of leading elements are independent. Nor can we eliminate $z_{0}^{n}, z_{1}^{n}, \ldots, z_{n-1}^{n}$, as that would make $z_{n}^{n}$ a linear function of $z$ 's with lower index, and $z_{i}^{i}$ also, by taking the $(n-i)$ th $y$-derivate; so that $z_{i}^{i}=0$, which is contrary to the hypothesis $a_{i}^{i} \neq 0 .{ }^{*}$ We here use the fact that every $z$ with index $<i$ vanishes, not assuming, however, that it is a consequence of the one-set $\Sigma \alpha_{q}^{p} \boldsymbol{z}_{q}^{p}=0$. Now the coefficients of $z_{0}^{n}, z_{1}^{n}, \ldots, z_{n-1}^{n}$ are leading elements in the set of degree $n-1$. Hence the set of degree $n-1$ has as many rows, and if irregular has the same number of rows, as the set of degree $n$.

11. Two corresponding arrays are defined as those which have the same number of columns and are such that the sum of the products of the elements of any row in one and any row in the other is zero. When each array consists of independent rows we shall call them corresponding independent arrays. It is clear that if the whole number of rows of two corresponding independent arrays is equal to the number of columns, each array represents the complete solution of the equations given by the other array; from which it follows also that the whole number of rows in the two arrays cannot exceed the number of columns in either array.

The modified $\zeta$ and $z$ arrays are corresponding independent arrays, and so also are the arrays of leading elements of any two sets of the same degree. Hence, to prove that the $z$ array gives the complete solution of the $\zeta$ equations, it has only to be shown that the leading elements of any two sets of the same degree together form a square array; for then the whole $\zeta$ and $z$ arrays also form a square array. This amounts to proving that if the set of degree $n$ in the $\zeta$ array is regular, then the set of degree $n-1$ in the $z$ array is regular. We shall prove this for a particular example, sufficiently complicated to make the general reasoning clear.

12. Suppose that $i=5$, and that the sets of degree $5,6,9,11,12$ of the $\zeta$ array are regular; then we have to prove that the sets of degree $4,5,8,10,11$ of the $z$ array are regular. We know that the last is regular, since by hypothesis, the $\alpha$ 's with limit index 11 do not vanish. We shall assume that the sets of degree 10 and 8 are regular also, in which case the sets of degree 9, 7,6 must be irregular, and prove that the sets of degree 5 and 4 are regular; this will include all the steps of the proof for the sets of degree 10 and 8 . The $\zeta$ and $z$ arrays are exhibited by the following table:

* This proof is taken from $T \S 7$; but is simplified by reason of the assumption $a_{i}^{i} \neq 0$. 


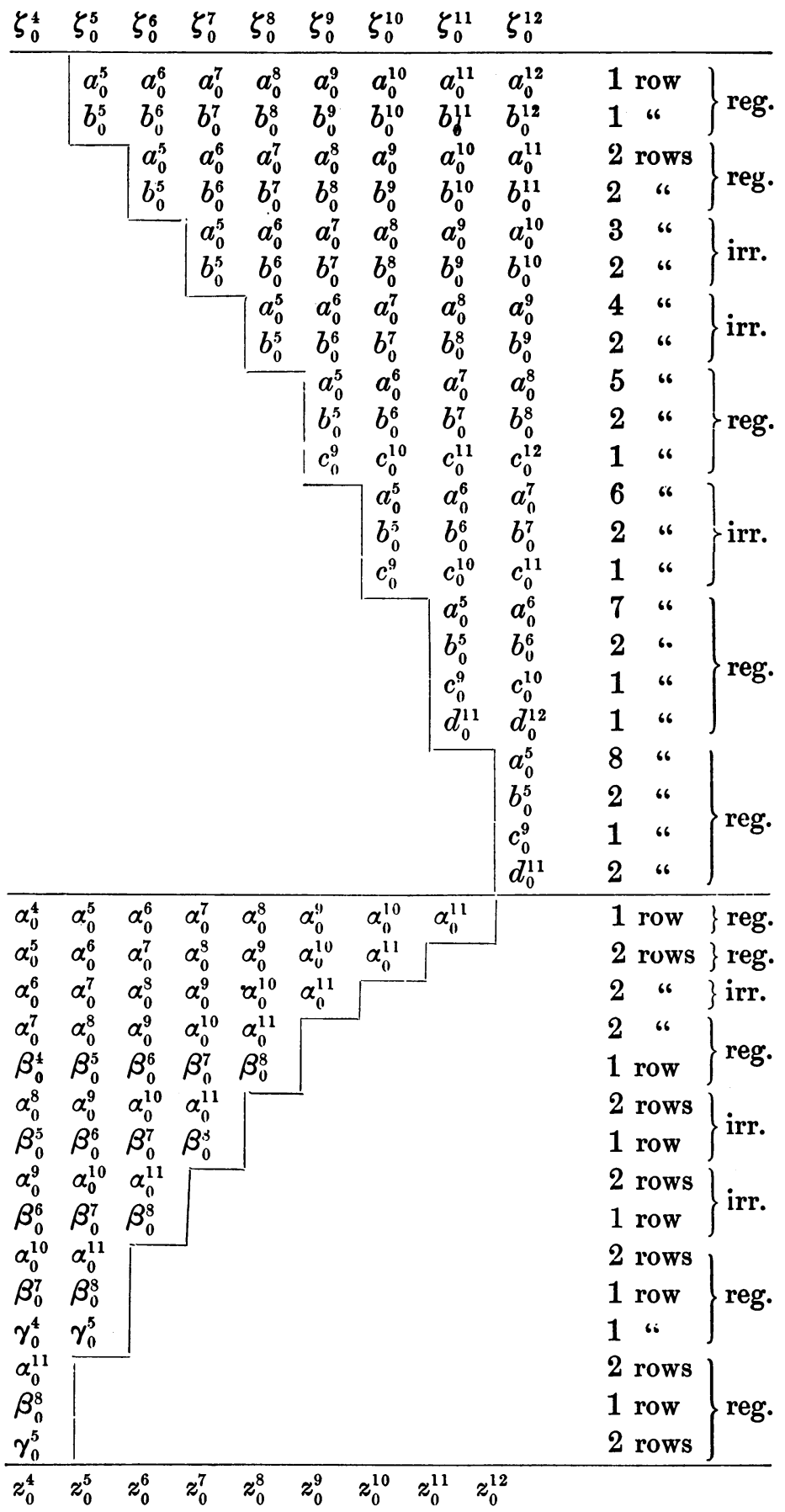

Only the merest outline of the arrays is given; but the filling up will be 
understood from what has been already said. Thus the leading elements of the third set of the upper array and fourth set of the lower array are respectively

$$
\begin{array}{lllllllllllllllllll}
a_{0}^{5} & a_{1}^{5} & a_{2}^{5} & a_{3}^{5} & a_{4}^{5} & a_{5}^{5} & 0 & 0 & \text { and } & \alpha_{0}^{11} & \alpha_{1}^{11} & \alpha_{2}^{11} & \alpha_{3}^{11} & \alpha_{4}^{11} & \alpha_{5}^{11} & \alpha_{8}^{11} & \alpha_{7}^{11} & \alpha_{8}^{11} \\
\mathbf{0} & a_{0}^{5} & a_{1}^{5} & a_{2}^{5} & a_{3}^{5} & a_{4}^{5} & a_{5}^{5} & 0 & & \alpha_{1}^{11} & \alpha_{2}^{11} & \alpha_{3}^{11} & \alpha_{4}^{11} & \alpha_{5}^{11} & \alpha_{6}^{11} & \alpha_{7}^{11} & \alpha_{8}^{11} & \alpha_{9}^{11} \\
\mathbf{0} & 0 & a_{0}^{5} & a_{1}^{5} & a_{2}^{5} & a_{3}^{5} & a_{4}^{5} & a_{5}^{5} & & \beta_{0}^{8} & \beta_{1}^{8} & \beta_{2}^{8} & \beta_{3}^{8} & \beta_{4}^{8} & \beta_{5}^{8} & \beta_{6}^{8} & \beta_{7}^{8} & \beta_{8}^{8} \\
b_{0}^{5} & b_{1}^{5} & b_{2}^{5} & b_{3}^{5} & b_{4}^{5} & b_{5}^{5} & 0 & 0 & & & & & & & & & & \\
\mathbf{0} & b_{0}^{5} & b_{1}^{5} & b_{2}^{5} & b_{3}^{5} & b_{4}^{5} & b_{5}^{5} & 0 & & & & & & & & & &
\end{array}
$$

A curious property of the $z$ array, which we do not stay to prove, is that the determinants formed by consecutive columns of the leading elements of any set which is one degree higher than an irregular set are in geometrical progression. The connected property of the $\zeta$ array is not expressed so simply.

13. We have to prove that the set of degree 5 in the $z$ array is regular, i. e., has 4 independent rows. If it is not regular, then, by joining it on to the two preceding irregular sets, we see that the following array must vanish :

$$
\begin{array}{lllllllll}
\alpha_{0}^{9} & \cdots & \alpha_{5}^{9} & \alpha_{0}^{10} & \cdots & \alpha_{6}^{10} & \alpha_{0}^{11} & \cdots & \alpha_{7}^{11} \\
\alpha_{1}^{9} & \cdots & \alpha_{6}^{9} & \alpha_{1}^{10} & \cdots & \alpha_{7}^{10} & \alpha_{1}^{11} & \cdots & \alpha_{8}^{11} \\
\beta_{0}^{6} & \cdots & \beta_{5}^{6} & \beta_{0}^{7} & \cdots & \beta_{6}^{7} & \beta_{0}^{8} & \cdots & \beta_{7}^{8} \\
\beta_{1}^{6} & \cdots & \beta_{6}^{6} & \beta_{1}^{7} & \cdots & \beta_{7}^{7} & \beta_{1}^{8} & \cdots & \beta_{8}^{8} \\
\alpha_{0}^{10} & \cdots & \alpha_{5}^{10} & \alpha_{0}^{11} & \cdots & \alpha_{6}^{11} & & & \\
\alpha_{1}^{10} & \cdots & \alpha_{6}^{10} & \alpha_{1}^{11} & \cdots & \alpha_{7}^{11} & & & \\
\beta_{0}^{7} & \cdots & \beta_{5}^{7} & \beta_{0}^{8} & \cdots & \beta_{6}^{8} & & & \\
\alpha_{0}^{11} & \cdots & \alpha_{5}^{11} & & & & & & \\
\alpha_{1}^{11} & \cdots & \alpha_{6}^{11} & & & & & & \\
\beta_{0}^{8} & \cdots & \beta_{5}^{3} & & & & & &
\end{array}
$$

The 4th row here is reinserted, since it belongs to the complete $z$ array, and gives rise to the row $\gamma$ in the set of degree 5 . The other 9 rows are independent, as already proved, so that the 4th row is dependent on the other 9 , on the supposition that the array vanishes. We may then add further columns to the array, so that the new array also vanishes, by properly choosing the values of the elements added in the 4 th row. We shall add three such columns, containing three new elements $\beta_{7}^{6}, \beta_{8}^{7}, \beta_{9}^{8}$ in the 4 th row. Then by comparing the sets of degree $6,7,8$ in the $\zeta$ and $z$ arrays the following are seen to be corresponding arrays, the 4 th row of the lower array being added, since it is dependent on the others : 


$$
\begin{aligned}
& \begin{array}{lllllll}
a_{0}^{5} & \ldots & a_{0}^{6} & \ldots & a_{0}^{7} & \ldots & 2 \text { rows }
\end{array} \\
& \begin{array}{lllllll}
b_{0}^{5} & \ldots & b_{0}^{6} & \ldots & b_{0}^{7} & \ldots & 2
\end{array} \\
& \begin{array}{llllll}
a_{0}^{5} & \ldots & a_{0}^{6} & \ldots & 3 &
\end{array} \\
& b_{0}^{5} \quad \ldots \quad b_{0}^{6} \quad \ldots \quad 2 \text { “ } \\
& \begin{array}{cccccccccc} 
& & & & & a_{0}^{5} & \cdots & & \\
& & & & & \\
& & & & & & \\
& & & & & 2 & & \\
\hline \alpha_{0}^{9} & \cdots & \alpha_{6}^{9} & \alpha_{0}^{10} & \cdots & \alpha_{7}^{10} & \alpha_{0}^{11} & \cdots & \alpha_{8}^{11} &
\end{array} \\
& \begin{array}{lllllllll}
\alpha_{1}^{9} & \ldots & \alpha_{7}^{9} & \alpha_{1}^{10} & \ldots & \alpha_{8}^{10} & \alpha_{1}^{11} & \ldots & \alpha_{9}^{11}
\end{array} \\
& \begin{array}{lllllllll}
\beta_{0}^{6} & \cdots & \beta_{6}^{6} & \beta_{0}^{7} & \cdots & \beta_{7}^{7} & \beta_{0}^{8} & \cdots & \beta_{8}^{8}
\end{array} \\
& \begin{array}{lllllllll}
\beta_{1}^{6} & \cdots & \beta_{7}^{6} & \beta_{1}^{7} & \cdots & \beta_{8}^{7} & \beta_{1}^{8} & \cdots & \beta_{9}^{8}
\end{array} \\
& \begin{array}{llllll}
\alpha_{0}^{10} & \cdots & \alpha_{6}^{10} & \alpha_{0}^{11} & \cdots & \alpha_{7}^{11}
\end{array} \\
& \begin{array}{llllll}
\alpha_{1}^{10} & \cdots & \alpha_{7}^{10} & \alpha_{1}^{11} & \cdots & \alpha_{8}^{11}
\end{array} \\
& \begin{array}{llllll}
\beta_{0}^{7} & \cdots & \beta_{6}^{7} & \beta_{0}^{8} & \cdots & \beta_{7}^{8}
\end{array} \\
& \alpha_{0}^{11} \quad \cdots \quad \alpha_{6}^{11} \\
& \alpha_{1}^{11} \quad \cdots \quad \alpha_{7}^{11} \\
& \beta_{0}^{8} \quad \cdots \quad \beta_{6}^{8}
\end{aligned}
$$

Hence the following arrays also correspond :

$$
\begin{aligned}
& \begin{array}{lllllll}
a_{0}^{5} & \ldots & a_{0}^{8} & \ldots & a_{0}^{7} & \ldots & 3 \text { rows }
\end{array} \\
& \begin{array}{llllllll}
b_{0}^{5} & \ldots & b_{0}^{6} & \ldots & b_{0}^{7} & \ldots & 3 &
\end{array} \\
& \begin{array}{llllll}
a_{0}^{5} & \ldots & a_{0}^{6} & \ldots & 4 &
\end{array} \\
& b_{0}^{5} \quad \cdots \quad b_{0}^{6} \quad \ldots \quad \quad 2 \quad 6 \\
& a_{0}^{5} \quad \cdots \quad 5 \quad 6 \\
& \begin{array}{llllllllll} 
& & & \multicolumn{1}{c}{b_{0}^{5}} & \cdots & 2 & 26 \\
\hline \alpha_{0}^{9} & \cdots & \alpha_{7}^{9} & \alpha_{0}^{10} & \cdots & \alpha_{8}^{10} & \alpha_{0}^{11} & \cdots & \alpha_{9}^{11}
\end{array} \\
& \begin{array}{lllllllll}
\alpha_{1}^{9} & \ldots & \alpha_{3}^{9} & \alpha_{1}^{10} & \ldots & \alpha_{9}^{10} & \alpha_{1}^{11} & \ldots & \alpha_{10}^{11}
\end{array} \\
& \begin{array}{lllllllll}
\beta_{0}^{6} & \cdots & \beta_{7}^{6} & \beta_{0}^{7} & \cdots & \beta_{8}^{7} & \beta_{0}^{8} & \cdots & \beta_{9}^{8}
\end{array} \\
& \begin{array}{llllll}
\alpha_{0}^{10} & \cdots & \alpha_{7}^{10} & \alpha_{0}^{11} & \cdots & \alpha_{8}^{11}
\end{array} \\
& \begin{array}{llllll}
\alpha_{1}^{10} & \cdots & \alpha_{8}^{10} & \alpha_{1}^{11} & \cdots & \alpha_{9}^{11}
\end{array} \\
& \begin{array}{llllll}
\beta_{0}^{7} & \cdots & \beta_{7}^{7} & \beta_{0}^{8} & \cdots & \beta_{8}^{8}
\end{array} \\
& \alpha_{0}^{11} \quad \ldots \quad \alpha_{7}^{11} \\
& \alpha_{1}^{11} \quad \ldots \quad \alpha_{8}^{11} \\
& \begin{array}{lll}
\beta_{0}^{8} & \ldots & \beta_{7}^{8}
\end{array}
\end{aligned}
$$


Here the only row not given by the sets of degree 7, 8, 9 of the table is the third row of the lower array, which takes the place of the $3 \mathrm{~d}$ and 4 th rows of the lower array in the previous step. Both arrays consist of independent rows, the last row of the 1st set in the upper array being equivalent to a row $c$ in the $3 \mathrm{~d}$ set. But the number of rows in the two arrays combined is greater than the number of columns, which is impossible. The set of degree 5 in the $z$ array must therefore have 4 independent rows, and is regular.

Again, the set of degree 4 of the $z$ array is regular; for the first 4 rows are independent $(\S 10)$, and, if the 5 th row is dependent on them, we can add an element $\gamma_{6}^{5}$ such that the array

$$
\begin{aligned}
& \alpha_{0}^{11} \cdots \alpha_{5}^{11} \\
& \alpha_{1}^{11} \cdots \alpha_{6}^{11} \\
& \beta_{0}^{3} \cdots \beta_{5}^{3} \\
& \gamma_{0}^{5} \cdots \gamma_{5}^{5} \\
& \gamma_{1}^{5} \cdots \gamma_{6}^{5}
\end{aligned}
$$

vanishes. To this corresponds the array

$$
\begin{aligned}
& a_{0}^{5} \cdots a_{5}^{5} \\
& b_{0}^{5} \cdots b_{5}^{5} ;
\end{aligned}
$$

hence the arrays

$$
\begin{aligned}
& a_{0}^{5} \cdots \quad 2 \text { rows } \\
& \frac{b_{0}^{5} \cdots \quad 2 \text { rows }}{\alpha_{0}^{11} \cdots \alpha_{6}^{11}} \\
& \alpha_{1}^{11} \cdots \alpha_{7}^{11} \\
& \beta_{0}^{8} \cdots \beta_{6}^{8} \\
& \gamma_{0}^{5} \cdots \gamma_{6}^{5}
\end{aligned}
$$

correspond; which is impossible, for the same reason as before.

The above reasoning, although applied to a particular example, is perfectly general, and shows that the general solution of any system of $\zeta$ equations, which includes $i$ regular sets of equations, is $\zeta_{q}^{p}=\Sigma \lambda_{s}^{r} \alpha_{q+s}^{p+r}$, where $\zeta_{q}^{p}=\alpha_{q}^{p}$ is a particular solution. From this the one-set theorem follows, as in $\S 8$ (vi).

14. The converse theorem that the complete solution of any one-set system of equations is expressible by means of two particular solutions $a, b\left(\right.$ viz. $\left.z_{q}^{p}=\Sigma \lambda_{s}^{r} a_{q-s}^{p-r}+\Sigma \mu_{s}^{r} b_{q-s}^{p-r}\right)$ is proved in a similar way. Taking the 
example already considered, we suppose the $z$ array given, and have to prove that there are two particular solutions $a, b$ of the $z$ equations such that, if the $\zeta$ array be formed for the series $a, b$, the set of degree $n$ in the $\zeta$ array is regular if the set of degree $n-1$ in the $z$ array is regular. We assume that the $z$ equations do not require $z_{5}^{5}$ to vanish (footnote $\S 7$ ). The series $a$ is then any solution in which $a_{5}^{5} \neq 0$, and the series $b$ is any other solution which supplies an additional independent row in the earliest set of the $\zeta$ array where it happens to be needed. In the example considered this is the first set, so that a suitable choice for the solution $b$ would be any one in which $b_{5}^{5}=0$, provided $b_{0}^{5}, b_{1}^{5}, b_{2}^{5}, b_{3}^{5}, b_{4}^{5}$ do not all vanish.

If the set of degree 6 in the $\zeta$ array were not then regular, $\gamma_{6}^{5}$ could be so chosen that the.two following arrays correspond:

$$
\begin{array}{lll}
a_{0}^{5} & \cdots & 2 \text { rows } \\
b_{0}^{5} \cdots & 2 \text { rows } \\
\hline \alpha_{0}^{11} \cdots \alpha_{6}^{11} & \\
\alpha_{1}^{11} \cdots \alpha_{7}^{11} & \\
\beta_{0}^{3} & \cdots \beta_{6}^{8} & \\
\gamma_{0}^{5} & \cdots & \gamma_{6}^{5}
\end{array}
$$

The $\gamma$ row is added by comparing the sets of degree 5 , assuming the $2 \mathrm{~d} b$ row dependent. From this it follows that the two independent arrays

$$
\begin{array}{ll}
a_{0}^{5} \cdots & 1 \text { row } \\
b_{0}^{5} \cdots & 1 \text { row } \\
\hline \alpha_{0}^{11} \cdots \alpha_{5}^{11} & \\
\alpha_{1}^{11} \cdots \alpha_{6}^{11} & \\
\beta_{0}^{8} \cdots \beta_{5}^{8} & \\
\gamma_{0}^{5} \cdots \gamma_{5}^{5} & \\
\gamma_{1}^{5} \cdots \gamma_{6}^{5} &
\end{array}
$$

correspond, which is impossible. Hence the set of degree 6 in the $\zeta$ array is regular. Similarly the rest of the proof of the direct theorem can be reversed; and it follows that the sets of degree 9,11, 12 in the $\zeta$ array are regular. This proves the converse theorem. The one-set theorem is, I believe, true for any number of dimensions; but the converse is only true for two dimensions. 
The irregularities of a one-set.

15. The diagram $(T \S 32)$ of the equations of the one-set of $\S 12$ above is

$$
\begin{array}{l|lllll}
z^{11} & E_{0}^{0} & & & & \\
z^{10} & E_{0}^{1} & E_{1}^{1} & & & \\
z^{9} & E_{0}^{2} & E_{1}^{2} & & & \\
z^{8} & E_{0}^{3} & E_{1}^{3} & F_{0}^{0} & & \\
z^{7} & E_{0}^{4} & E_{1}^{4} & F_{0}^{1} & & \\
z^{6} & E_{0}^{5} & E_{1}^{5} & F_{0}^{2} & & \\
z^{5} & E_{0}^{6} & E_{1}^{6} & F_{0}^{3} & G_{0}^{0} & \\
z^{4} & E_{0}^{7} & E_{1:}^{7} & F_{0}^{4} & G_{0}^{1} & G_{1}^{1},
\end{array}
$$

where $E_{0}^{0}=\Sigma \alpha_{q}^{p} z_{q}^{p}, F_{0}^{0}=\Sigma \beta_{q}^{p} z_{q}^{p}, G_{0}^{0}=\Sigma \gamma_{q}^{p} z_{q}^{p}$. This diagram shows the irregularities in the simplest way. There are three irregularities altogether, one before $F_{0}^{0}$ appears, and two consecutive ones before $G_{0}^{0}$ appears. Consecutive irregularities can be considered together, but separated irregularities must be considered by themselves in the order in which they appear.

It is clear that $F_{0}^{0}$ replaces $E_{2}^{2}$, and is equal to $E_{2}^{2}$ modified by $E$ 's which occur in the first two columns, viz.,

$$
F_{0}^{0}=f_{0}^{2} E_{0}^{2}+f_{1}^{2} E_{1}^{2}+f_{2}^{2} E_{2}^{2}, \quad \text { where } \quad f_{2}^{2}=1 .
$$

We shall say that $x^{p-q} y^{q}$ corresponds to $E_{q}^{p}$, so that the polynomial which corresponds to $F_{0}^{0}$ is

$$
F=f_{0}^{2} x^{2}+f_{1}^{2} x y+f_{2}^{2} y^{2} .
$$

Again $G_{0}^{0}$ is $F_{1}^{1}\left(\right.$ or $E_{3}^{3}$ ) modified, viz.,

$$
G_{0}^{0}=\left(F_{0}^{1}, F_{1}^{1}, F_{0}^{2}, E_{0}^{4}, E_{1}^{4}, E_{0}^{5}, E_{1}^{5}\right)^{(1)}=g_{0}^{3} E_{0}^{3}+\cdots,
$$

and to $G_{0}^{0}$ corresponds a series $g\left(g_{3}^{3}=1\right)$, and polynomial $G$, viz., $G=g_{0}^{3} x^{3}+g_{1}^{3} x^{2} y+g_{2}^{3} x y^{2}+g_{3}^{3} y^{3}+g_{0}^{4} x^{4}+g_{1}^{4} x^{3} y+g_{2}^{4} x^{2} y^{2}+g_{0}^{5} x^{5}+g_{1}^{5} x^{4} y$.

Also, from the first expression for $G_{0}^{0}$ above, since $x F, y F$ correspond to $F_{0}^{1}$, $F_{1}^{1}$, we have

$$
G=F u_{1}+P_{4},
$$

where $u_{n}$ is a homogeneous polynomial in $x, y$ of degree $n$, and $P_{n}$ denotes a polynomial, or power series, whose lowest terms are of degree $n$.

16. The relation $f_{0}^{2} E_{0}^{2}+f_{1}^{2} E_{1}^{2}+f_{2}^{2} E_{2}^{2}=F_{0}^{0}$, where $F_{0}^{0}$ is one degree lower in $z$ 's than $E_{0}^{2}, E_{1}^{2}, E_{2}^{2}$, shows that the following arrays correspond: 


\begin{tabular}{l|llllll}
\multicolumn{1}{l}{$z_{0}^{5}$} & $z_{1}^{5}$ & $z_{2}^{5}$ & $z_{3}^{5}$ & $z_{4}^{5}$ & $z_{5}^{5}$ \\
\cline { 2 - 7 }$f_{0}^{2}$ & $\alpha_{0}^{11}$ & $\alpha_{1}^{11}$ & $\alpha_{2}^{11}$ & $\alpha_{3}^{11}$ & $\alpha_{4}^{11}$ & $\alpha_{5}^{11}$ \\
$f_{1}^{2}$ & $\alpha_{1}^{11}$ & $\alpha_{2}^{11}$ & $\alpha_{3}^{11}$ & $\alpha_{4}^{11}$ & $\alpha_{5}^{11}$ & $\alpha_{6}^{11}$ \\
$f_{2}^{2}$ & $\alpha_{2}^{11}$ & $\alpha_{3}^{11}$ & $\alpha_{4}^{11}$ & $\alpha_{5}^{11}$ & $\alpha_{6}^{11}$ & $\alpha_{7}^{11}$ \\
\cline { 2 - 7 } & $f_{0}^{2}$ & $f_{1}^{2}$ & $f_{2}^{2}$ & & & \\
$m$ & & $f_{0}^{2}$ & $f_{1}^{2}$ & $f_{2}^{2}$ & & \\
$n$ & & & $f_{0}^{2}$ & $f_{1}^{2}$ & $f_{2}^{2}$ & \\
$r$ & & & & $f_{0}^{2}$ & $f_{1}^{2}$ & $f_{2}^{2}$ \\
\hline & & & & & &
\end{tabular}

Hence the lower array gives a complete solution for $z_{0}^{5}, \ldots, z_{5}^{5}$, so that $z_{0}^{5} x^{5}+z_{1}^{5} x^{4} y+\cdots+z_{5}^{5} y^{5}$

$$
=\left(f_{0}^{2} x^{2}+f_{1}^{2} x y+f_{2}^{2} y^{2}\right)\left(l x^{3}+m x^{2} y+n x y^{2}+r y^{3}\right)=F u_{3}
$$

or, if $S=\Sigma z_{q}^{p} x^{p-q} y^{q}$ is the general polynomial which satisfies the one-set,

Again, from the relation

$$
S=F u_{3}+P_{6} \text {. }
$$

$$
g_{0}^{3} E_{0}^{3}+\cdots+g_{3}^{3} E_{3}^{3}+g_{0}^{4} E_{0}^{4}+g_{1}^{4} E_{1}^{4}+g_{2}^{4} E_{2}^{4}+g_{0}^{5} E_{0}^{5}+g_{1}^{5} E_{1}^{5}=G_{0}^{0},
$$

combined with the former relation, we see that the two following arrays correspond:

$$
\begin{aligned}
& z_{0}^{5} z_{1}^{5} z_{2}^{5} z_{3}^{5} z_{4}^{5} z_{5}^{5} \quad z_{0}^{6} z_{1}^{6} z_{2}^{6} z_{3}^{6} z_{4}^{6} z_{5}^{6} z_{6}^{6} \quad z_{0}^{7} z_{1}^{7} z_{2}^{7} z_{3}^{7} z_{4}^{7} z_{5}^{7} z_{6}^{7} z_{7}^{7} \\
& \begin{array}{llllllllll}
g_{0}^{s} & \alpha_{0}^{9} & \cdots & \alpha_{5}^{9} & \alpha_{0}^{10} & \cdots & \alpha_{6}^{10} & \alpha_{0}^{11} & \cdots & \alpha_{7}^{11}
\end{array} \\
& \begin{array}{lllllllllll}
g_{1}^{3} & \alpha_{1}^{9} & \cdots & \alpha_{6}^{9} & \alpha_{1}^{10} & \ldots & \alpha_{7}^{10} & \alpha_{1}^{11} & \ldots & \alpha_{8}^{11}
\end{array} \\
& \begin{array}{lllllllllll}
g_{2}^{3} & \alpha_{2}^{9} & \cdots & \alpha_{7}^{9} & \alpha_{2}^{10} & \cdots & \alpha_{8}^{10} & \alpha_{2}^{11} & \cdots & \alpha_{9}^{11}
\end{array} \\
& \begin{array}{llllllllll}
g_{3}^{3} & \alpha_{3}^{9} & \cdots & \alpha_{8}^{9} & \alpha_{3}^{10} & \cdots & \alpha_{9}^{10} & \alpha_{3}^{11} & \cdots & \alpha_{10}^{11}
\end{array} \\
& \begin{array}{l|llllll}
g_{0}^{4} & \alpha_{0}^{10} & \cdots & \alpha_{5}^{10} & \alpha_{0}^{11} & \cdots & \alpha_{6}^{11}
\end{array} \\
& \begin{array}{l|llllll}
g_{1}^{4} & \alpha_{1}^{10} & \ldots & \alpha_{6}^{10} & \alpha_{1}^{11} & \ldots & \alpha_{7}^{11}
\end{array} \\
& \begin{array}{lllllll}
g_{2}^{4} & \alpha_{2}^{10} & \cdots & \alpha_{7}^{10} & \alpha_{2}^{11} & \cdots & \alpha_{8}^{11}
\end{array} \\
& g_{0}^{5} \quad \alpha_{0}^{11} \cdots \cdots \alpha_{5}^{11} \\
& \begin{array}{l|lll}
g_{1}^{5} & \alpha_{1}^{11} & \cdots & \alpha_{6}^{11}
\end{array} \\
& g_{0}^{3} g_{1}^{3} g_{2}^{3} g_{3}^{3} \quad g_{0}^{4} g_{1}^{4} g_{2}^{4} \quad g_{0}^{5} g_{1}^{5} \quad 3 \text { rows } \\
& g_{0}^{3} g_{1}^{3} g_{2}^{3} g_{3}^{3} \quad g_{0}^{4} g_{1}^{4} g_{2}^{4} \quad 4 \text { rows } \\
& f_{0}^{2} f_{1}^{2} f_{2}^{2} \quad 6 \text { rows. }
\end{aligned}
$$


Hence

$$
S=G\left(u_{2}+u_{3}\right)+F u_{5}+P_{8},
$$

since the upper array has all its rows independent except the 4 th, and consequently the lower array gives a complete solution for $z^{5}, z^{6}, z^{7}$.

Two results should be noted, which hold for any one-set: (i) $S$ is expressible by means of $F, G, \cdots$ up to and including terms of a degree equal to the index of $E$ in the last row of the diagram of the one-set; and (ii) the solution $S=G\left(u_{2}+u_{3}\right)+F u_{5}+P_{8}$ is the general solution of the $i$ equations of the one-set (and all their derivates) contained in the $i$ th line of the diagram when written in $E$ 's only, viz., in the present example,

$$
\begin{array}{lllll}
E_{0}^{0} & & & & \\
E_{0}^{1} & E_{1}^{1} & & & \\
E_{0}^{2} & E_{1}^{2} & E_{2}^{2} & & \\
E_{0}^{3} & E_{1}^{3} & E_{2}^{3} & E_{3}^{3} & \\
E_{0}^{4} & E_{1}^{4} & E_{2}^{4} & E_{3}^{4} & E_{4}^{4} \\
E_{0}^{5} & E_{1}^{5} & E_{2}^{5} & & \\
E_{0}^{6} & E_{1}^{6} & E_{2}^{6} & & \\
E_{0}^{7} & E_{1}^{7} . & & &
\end{array}
$$

The general solution of the whole one-set is obtained by applying to $u_{2}, u_{3}, u_{5}$, $P_{8}$ the conditions which arise from the equations contained in the first 4 (or $i-1)$ lines of this diagram. There are two equations $\left(F_{0}^{1}=0, G_{0}^{0}=0\right)$ which involve the coefficients of $u_{2}, u_{3}, u_{5}$ only, of which one $\left(G_{0}^{0}=0\right)$ involves the coefficients of $u_{2}$ only; the other 8 involve in addition the coefficients of $P_{8}$. The above form of the diagram is easily obtained from the original form by writing for the $F$ 's and $G$ 's their equivalent $E$ 's.

17. Take the still more complicated one-set whose diagram is

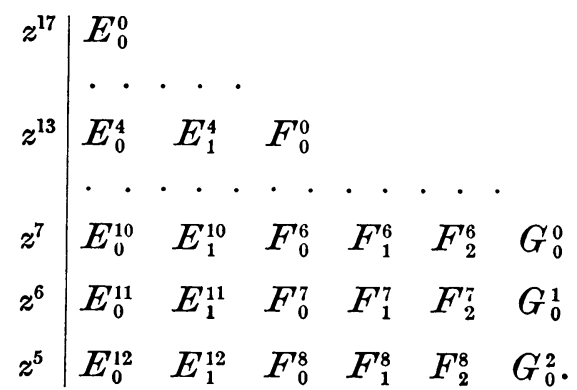

There are three groups of consecutive irregularities, to the third of which corresponds an expression $H_{0}^{0}$, containing no $z^{5}$ or $z^{6}$, where

$$
H_{0}^{0}=\left(G_{0}^{1}, G_{1}^{1}, G_{0}^{2}, F_{0}^{7}, F_{1}^{7}, F_{2}^{7}, F_{0}^{8}, F_{1}^{8}, F_{2}^{8}, E_{0}^{11}, E_{1}^{11}, E_{0}^{12}, E_{1}^{12}\right)^{(1)} \text {. }
$$


Since $F_{0}^{j}, G_{0}^{0}, H_{0}^{j}$ replace $E_{2}^{2}, E_{5}^{5}, E_{6}^{6}$ respectively, we have, as in $§ 16$,

$$
\begin{aligned}
& S=F\left(u_{4}+u_{5}\right)+P_{8}, \\
& S=G\left(u_{1}+u_{2}+u_{3}\right)+F\left(u_{7}+u_{8}\right)+P_{11}, \\
& S^{\prime}=H\left(u_{0}+u_{1}\right)+G\left(u_{3}+u_{4}+u_{5}\right)+F\left(u_{9}+u_{10}\right)+P_{13} .
\end{aligned}
$$

Also, from the relations,

we have

$$
\begin{aligned}
G_{0}^{0} & =\left(F_{0}^{3}, F_{1}^{3}, F_{2}^{3}, F_{3}^{3}, \cdots, E_{0}^{\gamma}, E_{1}^{7}, \cdots\right)^{(1)}, \\
H_{0}^{0} & =\left(G_{0}^{1}, G_{1}^{1}, G_{0}^{2}, F_{0}^{\gamma}, F_{1}^{\gamma}, F_{2}^{7}, \cdots, E_{0}^{11}, E_{1}^{11}, \cdots\right)^{(1)},
\end{aligned}
$$

$$
\begin{aligned}
& G=F\left(v_{3}+v_{4}\right)+P_{7}, \\
& H=G\left(v_{1}+v_{2}\right)+F\left(v_{7}+v_{8}\right)+P_{11}, \\
& H=G\left(v_{1}+v_{2}\right)+P_{9}, \quad \text { from (3), } \\
& H=F\left(v_{1} v_{3}+v_{5}\right)+P_{8}, \quad \text { from (2) and (3). }
\end{aligned}
$$

In (2) and (3) we may restrict $v_{4}$ to 3 terms, $x^{4}, x^{3} y, x^{2} y^{2}, v_{2}$ to 1 term, and $v_{7}, v_{8}$ each to 3 terms; but this has no special significance for what follows. None of the coefficients of $u_{0}, u_{1}, u_{3}, u_{4}, u_{5}, u_{9}, u_{10}$, in (1) can be dispensed with, although other forms than (1) might be adopted for $S$. The polynomial $F$ contains terms of degree 2 and $3, G$ contains terms of degree 5 to 9 , and $H$ contains terms of degree 6 to 12 . The relations (2) and (3) show that the addition of $H u_{2}$ or $G u_{6}$ would not alter the form of the right hand side of (1).

Geometrical interpretation. When the last of the polynomials $F, G, \ldots$ has all its tangents distinct the above results can be interpreted geometrically. The line $E_{0}^{1}, E_{1}^{1}$ of the diagram, being followed by 7 irregularities in all, indicates that the two branches of $S$ whose tangents are $f_{0}^{2} x^{2}+f_{1}^{2} x y+f_{2}^{2} y^{2}=0$ have contact of the 7 th order with two branches of $H$, i. e., they are fixed to the 7th order. The line $E_{0}^{6}, E_{1}^{6}, F_{0}^{2}, F_{1}^{2}, F_{2}^{2}$, being followed by 5 irregularities, indicates that 3 more branches of $S$, whose tangents are $v_{3}=0$, have contact of the 5 th order with three other branches of $H$; and the line $E_{0}^{10}, \ldots, G_{0}^{0}$, being followed by 2 irregularities, indicates that the last branch of $S$, whose tangent is $v_{1}=0$, has contact of the $2 \mathrm{~d}$ order with the last branch of $H$. In other words, all the 6 branches of $S$ are fixed to the $2 d$ order, 5 branches are fixed to the 5 th order, and 2 to the 7 th order.

To prove this, let $G^{\prime}, F^{\prime}$ denote the power series which divide $H$, and have the same tangents as $G, F$ respectively. Then from (4) $G=G^{\prime}+P_{8}$, and from (1) $S=G^{\prime} P_{1}+P_{11}$; hence 5 of the branches of $S$ have contact with $G^{\prime}$, and with $H$, of the 5 th order. Again, from (5), $F=F^{\prime}+P_{4}$, and from (3), $G\left(v_{1}+v_{2}\right)=F^{\prime}\left(v_{1} v_{3}+\cdots\right)+P_{11}$; hence $F^{\prime}$ has contact with $G$ of the 5 th order, i. e., $G=F^{\prime}\left(v_{3}+\cdots\right)+P_{10}$; hence, from (1), $S=F^{\prime} P_{4}+P_{13}$; i. e., two of the branches of $S$ have contact with $F^{\prime}$, and with $H$, of the 7 th order. 
This interpretation of the irregularities agrees with that suggested by Miss Scott in $T \S 32$. The interpretation does not apply if $H$ has any cusp or higher singularity at the origin.

18. The number of intersections. It follows from $\S 17$, when the last of the polynomials $F, G, \cdots$ has all its tangents distinct, that the whole intersection at the origin of any two curves which satisfy the one-set is at least equivalent to as many ordinary points as there are independent equations in the one-set. From the diagram we can choose 6 lines containing $1,2, \ldots, 6$ equations respectively, to which may be added the 15 equations $z^{p}=0$ when $p<5$ (the equations $z^{5}=0$ being included in the diagram). These make $6^{2}$ (or $i^{2}$ ) equations. There are still left in the diagram 2 lines containing 2 equations preceding the line containing $F_{0}^{0}, 3$ lines of 5 equations preceding $G_{0}^{0}$, and 2 lines of 6 equations after $G_{0}^{0}$; counting these by columns there are $2 \times 7+3 \times 5+1 \times 2$ equations, which equals the number of additional intersections of the two curves due to the contacts of their several branches, which they have in common with $H$. If the two curves had further contact the number of their intersections would exceed the number of independent equations of the one-set; but in this case it follows from what is proved below that the one-set would not then be the whole one-set of the two curves.

This result follows from the relations (1), (2), (3) on the supposition that all the tangents of $H$ are distinct. If $H$ had some or all of its tangents coincident, the number of the intersections would not thereby be diminished, although it is conceivable that it might be increased. Hence we may assume it to be always true that the number of intersections at the origin of any two curves which satisfy a given one-set is at least equal to the number of independent equations of the one-set.

From this it can be shown that the two numbers are exactly equal when the one-set is the whole one-set of the two curves. Choose two curves $C_{1}, C_{2}$ of degrees $l, m$, having a given one-set for their whole one-set ( $\$ 14)$, and such that their terms of highest degree have no factor in common. Let $S$ be a curve of degree $\geqq l+m-2$ with all its coefficients at disposal. By making $S$ satisfy the one-set, we have $S=C_{1} P_{1}+C_{2} P_{2}$. Also, by moving the origin in succession to each point of intersection of $C_{1}, C_{2}$, and making $S$ satisfy all the corresponding one-sets, $S$ takes the form $C_{1} P_{1}+C_{2} P_{2}$ for every point of intersection of $C_{1}, C_{2}$. Hence, by NoEthen's Theorem, $S=C_{1} S_{1}+C_{2} S_{2}$, where $C_{1} S_{1}$ and $C_{2} S_{2}$ are of the same degree as $S$, since the terms of degree $l, m$ in $C_{1}, C_{2}$ have no common factor. But the number of independent equations that must be satisfied by the coefficients of $S$ (of degree $\geqq l+m-2$ ) in order that it may be of the above form is known to be $l m$, which is also the number of ordinary points of intersection of $C_{1}, C_{2}$. Hence the equations of the onesets of $C_{1}, C_{2}$ for all their points of intersection are equivalent to the same 
number $(l m)$ of independent equations. This can only be true when the number of independent equations in each one-set is equal to (since it does not exceed) the corresponding number of ordinary intersections.

19. Another theorem of importance follows from $\S 17$. Any $t$-set contained in a one-set $E$ can be written in the form of the equations $\Sigma a_{q}^{p} E_{q}^{p}=0$, $\Sigma b_{q}^{p} E_{q}^{p}=0, \ldots$ with all their derivates $\Sigma a_{q}^{p} E_{q+m}^{p+l}=0, \ldots$. The theorem is that if the t-set includes all the equations in the first line of the diagram of $E$ which has the full complement of $i$ equations then any series which satisfies the one-set is expressible by means of the series $a, b, \ldots$, i. e.,

$$
S=A P+B Q+\cdots
$$

where $A=\Sigma a_{q}^{p} x^{p-q} y^{q}, \ldots$, and $P, Q, \ldots$ are power series. Also $P, Q, \ldots$ can all be chosen so as to vanish when $x=y=0$. Also, if $\Sigma a_{q}^{\prime p} E_{q}^{p}=0$ is any equation included in the t-set, then the series $a^{\prime}$ is expressible by means of ${ }^{\prime}$ the series $a, b, \ldots$.

We shall prove this for the ore-set of $\S 17$. The $t$-set then includes all the equations $E_{0}^{10}, E_{1}^{10}, F_{0}^{6}, F_{1}^{6}, F_{2}^{6}, G_{0}^{0}$. Any equation $\Sigma a_{q}^{\prime p} E_{q}^{p}=0$ included in the $t$-set is expressible identically in the form

$$
\Sigma \alpha_{q}^{\prime p} E_{q}^{p}=\Sigma \lambda_{s}^{r} \Sigma a_{q}^{p} E_{q+s}^{p+r}+\Sigma \mu_{s}^{r} \Sigma b_{q}^{p} E_{q+s}^{p+r}+\cdots,
$$

for $\Sigma a_{q}^{p} E_{q+\iota}^{p+r}$, etc., include all the independent expressions in $z$ involved in the equations of the $t$-set. This identity, however, is one in $z$ 's ; but we may equate coefficients of $E$ 's if we first add to the right hand side all expressions in $E$ 's which identically vanish, each with an undetermined multiplier. All such expressions, as well as several others which do not identically vanish, are included in $x$-derivates of $E_{0}^{10}, E_{1}^{10}, F_{0}^{6}, F_{1}^{6}, F_{2}^{6}$, and all derivates of $G_{0}^{0}$. Hence we obtain

$$
A^{\prime}=A P+B Q+\cdots+G P_{1}+F P_{7}+P_{11},
$$

where $P_{n}$ denotes as before a power series whose lowest terms are of degree $n$. It has to be shown that $G P_{1}+F P_{7}+P_{11}$ may be omitted from this identity. Since $E_{0}^{10}, E_{1}^{10}$ are included in the $t$-set and $E_{2}^{10}, E_{3}^{10}, \ldots, E_{10}^{10}$ are expressible in terms of these and $F^{\prime \prime}$ 's with index $\geqq 8$ (for $F_{0}^{0}$ replaces $E_{2}^{2}$ ) we may put $A^{\prime}$ equal to any one of $x^{10}, x^{9} y, \ldots, y^{10}$; hence $P_{11}$ may be omitted from the form for $A^{\prime}$. Again, since $F_{0}^{6}, F_{1}^{6}, F_{2}^{6}$ are included in the $t$-set, and $F_{3}^{6}, \ldots F_{6}^{6}$ are expressible in terms of these and $E$ 's with index $\geqq 10$ and $G$ 's with index $\geqq 3$, we may put $A^{\prime}$ equal to any one of the polynomials $x^{6} F, x^{5} y F, \cdots, y^{6} F^{\prime}$; from which it easily follows that $F P_{7}$ may be omitted in the form for $A^{\prime}$. Finally, since $G_{0}^{0}$ is included in the $t$-set, we may write $G$ for $A^{\prime}$; so that $G P_{1}$ may be omitted. Hence

$$
A^{\prime}=A P+B Q+\cdots,
$$

i. e., the series $a^{\prime}$ is expressible by means of the series $a, b, \ldots$

Also, since $(\S 17)$

$$
S=G\left(u_{1}+u_{2}+u_{3}\right)+F\left(u_{7}+u_{8}\right)+P_{11},
$$


and $G, F P_{6}, P_{10}$ are all of the form $A^{\prime}$, we have

$$
S=A P+B Q+\cdots,
$$

where $P, Q, \ldots$ are power series which all vanish when $x=y=0$. Hence also we have the following theorem, which will be used later:-

$A$ one-set $E$ can always be found containing any given $t$-set, such that if the t-set be expressed in any manner in the form $\Sigma a_{q}^{p} E_{q}^{p}=0, \Sigma b_{q}^{p} E_{q}^{p}=0, \cdots$, then any polynomial satisfying the one-set is of the form $A P+B Q+\cdots$, where $A=\Sigma a_{q}^{p} x^{p-q} y^{q}, \ldots$, and $P, Q, \ldots$ are power series which vanish when $x=y=0$.

Let $a^{\prime}, b^{\prime}, \ldots$ be the series, and $A^{\prime}, B^{\prime}, \ldots$ the corresponding polynomials, by means of which the general solution of the $t$-set can be expressed. Let $A^{\prime}$ have terms of as low degree $i$ as any one of the polynomials $A^{\prime}, B^{\prime}, \ldots$ Divide $A^{\prime}$ into all the other polynomials until remainders are obtained commencing with terms of as high degree as possible; these remainders may be taken as the polynomials $B^{\prime}, C^{\prime}, \ldots$ Let $B^{\prime}$ have terms of as low degree $j(\geqq i)$ as any one of $B^{\prime}, C^{\prime}, \ldots$ Then the one-set of $A^{\prime}, B^{\prime}$ satisfies the conditions. This one-set contains the $t$-set; each of the lines in its diagram corresponding to $z^{i-1}, \ldots, z^{j-1}$ contains $i$ equations, and the line corresponding to $z^{j}$ contains only $i-1$ equations. Also the equations in the line corresponding to $z^{j-1}$ gives all the one-sets of degree $j-1$ satisfied by the series $a^{\prime}$; and these one-sets are all satisfied by each of the series $a^{\prime}, b^{\prime}, \ldots$ (since every $b^{\prime}, c^{\prime}, \ldots$ with index $<j$ vanishes), and are therefore all included in the $t$-set. Hence the theorem follows.

It is not necessary for this theorem that the series $a, b, \ldots$ should be independent, or limited in number; nor is it necessary that the one-set should be restricted to the choice that has been made. It is proved in $\$ \S 22,23$ that the one-set $E$ may be chosen as the one-set of any pair of the series $a^{\prime}, b^{\prime}, \ldots$, provided these are all independent.

20 . In the case where the $\zeta$ equations of two series $a, b$ include only $k$ regular sets altogether, where $k<i$, the following array will vanish, however far it may be carried :

$$
\begin{array}{cccccccc}
a_{0}^{i} & \cdots & a_{0}^{i+1} & \ldots & a_{0}^{i+2} & \ldots & k+1 \text { rows } \\
b_{0}^{i} & \ldots & b_{0}^{i+1} & \ldots & b_{0}^{i+2} & \ldots & k+1 & \text { “ } \\
& & a_{0}^{i} & \ldots & a_{0}^{i+1} & \ldots & k+2 & \text { “ } \\
& & b_{0}^{i} & \cdots & b_{0}^{i+1} & \ldots & k & \text { “ } \\
& & & & a_{0}^{i} & \ldots & k+3 & \text { “ } \\
& & & & b_{0}^{i} & \ldots & k & \text { “ }
\end{array}
$$


there being only $k$ rows $b$ in each set except the first. For if the rows of this array were all independent, and we modify it so that the leading elements in each set may be independent, we must come sometime to a set containing $k+1$ rows in addition to the rows $a$, which is contrary to the hypothesis. Hence the whole last row of the 1st set is dependent on the other rows of the array. We may border the array by a row of arbitrary elements, and a column which is not arbitrary, viz.,

$$
\begin{gathered}
\rho_{0}^{k}, \ldots, \rho_{k}^{k}, \sigma_{0}^{k}, \ldots, \sigma_{k}^{k}, \rho_{0}^{k+1}, \ldots, \rho_{k+1}^{k+1}, \sigma_{0}^{k+1}, \ldots, \sigma_{k-1}^{k+1}, \\
\rho_{0}^{k+2}, \cdots, \rho_{k+2}^{k+2}, \sigma_{0}^{k+2}, \ldots, \sigma_{k-1}^{k+2}, \cdots,
\end{gathered}
$$

in which $\sigma_{k}^{k}$ is not zero, and take the sum of every element in the array multiplied by the two corresponding elements in the border to be zero. Choosing the elements of the border row to be

$$
x^{i+k}, x^{i+k-1} y, \cdots, y^{i+k}, x^{i+k+1}, x^{i+k} y, \cdots, y^{i+k+1}, x^{i+k+2}, \cdots,
$$

we obtain the identity

or

$$
\rho_{0}^{k} x^{k} C_{1}+\rho_{1}^{k} \dot{x}^{k-1} y C_{1}+\cdots+\sigma_{0}^{k} x^{k} C_{2}+\sigma_{1}^{k} x^{k-1} y C_{2}+\cdots=0,
$$

$$
C_{1} P_{1}+C_{2} P_{2}=0
$$

where $P_{1}, P_{2}$ are power series in which terms of order $k$ are present, since $\sigma_{k}^{k} \neq 0$. Hence $C_{1}, C_{2}$ have a common factor with a multiple point at least of order $i-k$ at the origin; $*$ for if all the common factors of $P_{2}, P_{1}$ which vanish at the origin be divided out, $C_{1}$ and $C_{2}$ will be divisible by the factors which remain, and the quotients will be identical, except in sign.

\section{Independence of one-sets and of series.}

21. A t-set system of equations is not equivalent to any but a t-set system.

It will be sufficient to prove this for a 3 -set, $E=E^{\prime}=E^{\prime \prime}=0$. In the first place no identical relation of the form

$$
\Sigma \lambda_{s}^{r} E_{s}^{r}+\Sigma \lambda_{s}^{\prime r} E_{s}^{\prime r}+\Sigma \lambda_{s}^{\prime r} E_{s}^{\prime \prime r}=0
$$

can exist, in which $\lambda_{0}^{0}, \lambda_{0}^{\prime 0}, \lambda_{0}^{\prime \prime 0}$ are not all zero. For if $\lambda_{c}^{0}=1$, then, by taking all the derivates of the identity we can find all the derivates of $E$, and finally $E$ itself, in terms of $E^{\prime}, E^{\prime \prime}$ and their derivates, so that the system is not a 3 set. Suppose then that the 3 -set is equivalent to a 2 -set $F^{\prime}=F^{\prime}=0$. Then $E, E^{\prime}, E^{\prime \prime}$ are expressible in terms of $F, F^{\prime}$ and their derivates, and also $F, F^{\prime}$ in terms of $E, E^{\prime}, E^{\prime \prime}$ and their derivates, i. e.,

* Brrry, On a case of divisibility, etc., Proceedings of the London Mathematical S ociety, vol. 30 (1899), p. 275. 


$$
\begin{aligned}
E & =\lambda F+\lambda^{\prime} F^{\prime}+\cdots+\lambda_{s}^{r} F_{s}^{r}+\lambda_{s}^{\prime r} F_{s}^{\prime r}+\cdots, \\
E^{\prime} & =\mu F+\mu^{\prime} F^{\prime}+\cdots, \\
E^{\prime \prime} & =\nu F+\nu^{\prime} F^{\prime}+\cdots, \\
F^{\prime} & =\rho E+\rho^{\prime} E^{\prime}+\rho^{\prime \prime} E^{\prime \prime}+\cdots, \\
F^{\prime} & =\sigma E+\sigma^{\prime} E^{\prime}+\sigma^{\prime \prime} E^{\prime \prime}+\cdots
\end{aligned}
$$

Substitute from the last two and their derivates in the first three, then

$$
\begin{aligned}
& E=\lambda\left(\rho E+\rho^{\prime} E^{\prime}+\rho^{\prime \prime} E^{\prime \prime}\right)+\lambda^{\prime}\left(\sigma E+\sigma^{\prime} E^{\prime}+\sigma^{\prime \prime} E^{\prime \prime}\right)+\cdots, \\
& E^{\prime}=\mu\left(\rho E+\rho^{\prime} E^{\prime}+\rho^{\prime \prime} E^{\prime \prime}\right)+\mu^{\prime}\left(\sigma E+\sigma^{\prime} E^{\prime}+\sigma^{\prime \prime} E^{\prime \prime}\right)+\cdots, \\
& E^{\prime \prime}=\nu\left(\rho E+\rho^{\prime} E^{\prime \prime}+\rho^{\prime \prime} E^{\prime \prime}\right)+\nu^{\prime}\left(\sigma E+\sigma^{\prime} E^{\prime}+\sigma^{\prime \prime} E^{\prime \prime}\right)+\cdots
\end{aligned}
$$

In the last three identities the coefficients of $E, E^{\prime}, E^{\prime \prime}$ vanish, from above; hence we have

$$
\left|\begin{array}{lll}
\lambda & \lambda^{\prime} & 0 \\
\mu & \mu^{\prime} & 0 \\
\nu & \nu^{\prime} & 0
\end{array}\right| \times\left|\begin{array}{ccc}
\rho & \sigma & 0 \\
\rho^{\prime} & \sigma^{\prime} & 0 \\
\rho^{\prime \prime} & \sigma^{\prime \prime} & 0
\end{array}\right|=\left|\begin{array}{lll}
1 & 0 & 0 \\
0 & 1 & 0 \\
0 & 0 & 1
\end{array}\right|=1,
$$

which is impossible. Hence it is clear that the 3 -set is not equivalent to any but a 3-set.

Any number of series $a, b, c, \cdots$ are said to be independent if no one is expressible by means of the rest. In such a case no relation of the form $\Sigma \alpha_{s}^{r} a_{q-s}^{p-r}+\Sigma \beta_{s}^{r} b_{q-s}^{p-r}+\cdots=0$ can exist for all values of $p, q(p \geqq q \geqq 0)$ fixed for one relation, unless $\alpha_{0}^{0}, \beta_{0}^{0}, \ldots$ all vanish. For the above relation gives an identity $A_{1} C_{1}+A_{2} C_{2}+\cdots=0$, where $A_{1}, A_{2}, \ldots, C_{1}, C_{2}, \cdots$ are the power series corresponding to the series $\alpha, \beta, \cdots, a, b, \ldots$, from which one of the power series $C_{1}, C_{2}, \ldots$ is expressible by means of the rest if one of the constant terms of $A_{1}, A_{2}, \ldots$, viz., $\alpha_{0}^{0}, \beta_{0}^{0}, \ldots$, does not vanish. From this it can be proved in the same way as above that $t+1$ independent series are not equivalent to any but $t+1$ independent series either in respect to the series which are expressible by means of them, or in respect to the one-sets which they satisfy.

\section{The t-set theorem.}

22. Consider all the one-sets satisfied by the $t^{\prime}+2$ given independent series $a^{\prime}, b^{\prime}, \ldots$ Let $E=\Sigma \alpha_{q}^{p} z_{q}^{p}$ be any one-set (e. g., the one-set of $a^{\prime}, b^{\prime}$, which is the choice we shall eventually make) containing all the one-sets. Then the general one-set contained in $E$ is

$$
\sum_{z_{q}^{\prime \prime}}^{\prime p} E_{q}^{p}=0
$$


In order that this and all its derivates $\sum_{z_{q}^{\prime}} E_{q+m}^{p+l}=0$ may be satisfied by the series $a^{\prime}$, we have

$$
\Sigma_{z_{q}^{\prime p}}^{\prime p} A_{q}^{\prime p}=0, \cdots, \Sigma_{q}^{\prime p} A_{q+m}^{\prime p+l}=0, \cdots,
$$

where $A_{q}^{\prime p}$ is the value of $E_{q}^{p}$ when the series $a^{\prime}$ is written for $z$. But

and similarly

$$
\begin{aligned}
\Sigma z_{q}^{\prime p} A_{q}^{\prime p} & =\Sigma z_{q}^{\prime p} \Sigma \alpha_{s}^{r}{a_{s-q}^{\prime r-p}}^{\prime-p}=\Sigma z_{q}^{\prime p} \Sigma \alpha_{s+q}^{r+p}{a_{s}^{\prime r}}^{r} \\
& =\Sigma a_{s}^{\prime r} \Sigma \alpha_{s+q}^{r+p} z_{q}^{\prime p}=\Sigma a_{s}^{\prime r} \Sigma \alpha_{q}^{p} z_{q-s}^{\prime p-r}=\Sigma{a_{s}^{\prime r}}^{\prime r} E_{s}^{\prime r},
\end{aligned}
$$

$$
\Sigma_{q}^{\prime p} A_{q+m}^{\prime p+l}=\Sigma a_{s}^{\prime r} E_{s+m}^{\prime r+l} .
$$

Hence the necessary and sufficient condition that the series $a^{\prime}$ may satisfy the one-set $\Sigma_{q}^{\prime p} E_{q}^{p}=0$ is that the series $z^{\prime}$ should satisfy the one-set $\Sigma{a_{s}^{\prime r}}^{r} E_{s}^{r}=0$, or $\Sigma a_{q}^{\prime p} E_{q}^{p}=0$; and in order that all the series $a^{\prime}, b^{\prime}, \ldots$ may satisfy the one-set $\Sigma z_{q}^{\prime p} E_{q}^{p}=0$, it is necessary and sufficient that the series $z^{\prime}$ should satisfy the $t^{\prime}+2$ one-sets $\Sigma a_{q}^{\prime p} E_{q}^{p}=0, \Sigma b_{q}^{\prime p} E_{q}^{p}=0, \cdots$

Let the general solution of these $t^{\prime}+2$ one-sets be expressible by the $t$ independent series $a, b, \ldots$; then expressing $z^{\prime}$ by the series $a, b, \ldots$, and substituting in $\Sigma_{q}^{\prime p} E_{q}^{p}=0$, we see that the $t$ one-sets

$$
\Sigma a_{q}^{p} E_{q}^{p}=0, \Sigma b_{q}^{p} E_{q}^{p}=0, \cdots
$$

make up all the one-sets which the given series $a^{\prime}, b^{\prime}, \ldots$ satisfy. Between these $t$ one-sets, and the $t^{\prime}+2$ one-sets

$$
\Sigma a_{q}^{\prime p} E_{q}^{p}=0, \Sigma b_{q}^{\prime p} E_{q}^{p}=0, \cdots \quad\left(t^{\prime}+2 \text { one-sets }\right),
$$

a reciprocal relation exists, viz., the complete solution of the $t$ one-sets is given by the $t^{\prime}+2$ independent series $a^{\prime}, b^{\prime}, \ldots$, and the complete solution of the $t^{\prime}+2$ one-sets is given by the $t$ independent series $a, b, \ldots$. Such mutually related systems are said to be residual with respect to the one-set $E .{ }^{*}$

* If $S$ is the general power series which satisfies the $t$ one-sets, then

$$
S=A^{\prime} P+B^{\prime} Q+\cdots,
$$

where $A^{\prime}, B^{\prime}, \cdots$ are the power series corresponding to the series $a^{\prime}, b^{\prime}, \cdots$, and $P, Q, \cdots$ are arbitrary power series. Further, in order that $S^{\prime}$ may be the general power series such that $S S^{\prime}$ satisfies the one-set, it is necessary and sufficient that $A^{\prime} S^{\prime}, B^{\prime} S^{\prime}, \cdots$ should satisfy the one-set, i. e., that $S^{\prime}$ should satisfy the $t^{\prime}+2$ one-sets, or that $S^{\prime}$ should be of the form

$$
S^{\prime}=A P+B Q+\cdots
$$

Thus, if $S, S^{\prime}$ are any power series satisfying the $t$ one-sets and $t^{\prime}+2$ one-sets respectively, then $S S^{\prime}$ satisfies the one-set. It is proved in $P \& 32$ that the number of independent equations in the $t$ one-sets added to the number of independent equations in the $t^{\prime}+2$ one-sets is equal to the number of independent equations in the one-set $E$. 
23. The $t^{\prime}+2$ one-sets $\Sigma{a^{\prime}}_{q}^{p} E_{q}^{p}=0$, etc., will be independent provided the series $h, k$ which determine the one-set $E$ are such that

$$
\begin{aligned}
& H=A^{\prime} P+B^{\prime} Q+\cdots, \\
& K=A^{\prime} P^{\prime}+B^{\prime} Q^{\prime}+\cdots,
\end{aligned}
$$

where $P, Q, \ldots, P^{\prime}, Q^{\prime}, \ldots$ are power series which all vanish when $x=y=0$. For the $t^{\prime}+1$ one-sets $\Sigma b_{q}^{\prime p} E_{q}^{p}=0, \Sigma c^{\prime}{ }_{q}^{p} E_{q}^{p}=0, \cdots$ are the equations which the series $z^{\prime}$ has to satisfy in order that the series $b^{\prime}, c^{\prime}, \ldots$ may satisfy the one-set $\Sigma z^{\prime}{ }_{q} E_{q}^{p}=0$; and if the one-set $\Sigma{a^{\prime \prime}}_{q} E_{q}^{p}=0$ is dependent on the remaining $t^{\prime}+1$ one-sets, the series $a^{\prime}$ must satisfy all the one-sets contained in $E$, viz., all the one-sets $\sum_{z^{\prime \prime}}{ }_{q} E_{q}^{p}=0$, which the series $b^{\prime}, c^{\prime}, \ldots$ satisfy, i. e., $a^{\prime}$ must satisfy all the one-sets which $h, k, b^{\prime}, c^{\prime}, \cdots$ satisfy. Hence $A^{\prime}$ must be expressible by means of $H, K, B^{\prime}, C^{\prime}, \ldots$ and therefore also by means of $B^{\prime}, C^{\prime}, \ldots$ alone, which is impossible, since the series $a^{\prime}, b^{\prime}, \ldots$ are independent.

Again, the $t^{\prime}+2$ one-sets cannot reduce to less than $t^{\prime}$ independent one-sets, and do reduce to a $t^{\prime}$-set if $H=A^{\prime}$ and $K=B^{\prime}$; for then $\Sigma a_{q}^{\prime p} E_{q}^{p}$ and $\Sigma{b^{\prime}}_{q}^{p} E_{q}^{p}$ vanish identically. If the first three of the $t^{\prime}+2$ one-sets were dependent on the rest, it would follow as above that the series $a^{\prime}, b^{\prime}, c^{\prime}$ would be expressible by means of $h, k$ and the remaining series, so that the $t^{\prime}+2$ independent series $a^{\prime}, b^{\prime}, \ldots$ would be equivalent to $t^{\prime}+1$ independent series only, which is impossible $(\S 20)$.

Similar properties hold for the $t$ one-sets $\Sigma a_{q}^{p} E_{q}^{p}=0$, etc., which form a definite or given system of equations, independent of $E$, since their solutions are the given series $a^{\prime}, b^{\prime}, \ldots$. Choose two solutions $h, k$ of the $t$ one-sets for determining the one-set $E$ as in $\S 18$, so that

$$
H=A P+B Q+\cdots, \quad K=A P^{\prime}+B Q^{\prime}+\cdots,
$$

where $P, Q, \ldots, P^{\prime}, Q^{\prime}, \ldots$ all vanish when $x=y=0$. In this case we may take $H, K$ to be $A^{\prime}, B^{\prime}$ respectively ; the $t$ one-sets are then independent, from above, i. e., they make a $t$-set, and the $t^{\prime}+2$ one-sets make a $t^{\prime}$-set. Hence to any $t$-set, determined by $t^{\prime}+2$ independent series, there corresponds a residual $t^{\prime}$-set determined by $t$ independent series. Thus, starting from the $t$-set, we can form the following chain of residues, to each of which we can assign the corresponding number of independent series,

$$
\begin{array}{llll}
t \text {-set } & t^{\prime} \text {-set } & (t-2) \text {-set } & \left(t^{\prime}-2\right) \text {-set } \\
\left(t^{\prime}+2\right) \text { series } & t \text { series } & t^{\prime} \text { series } & (t-2) \text { series }
\end{array} \text { etc. }
$$

If $t^{\prime}$ is odd we must come to a one-set, to which nothing but two series can correspond ( $\$ 14)$; and if $t^{\prime}$ is even, we must come to two series, to which noth- 
ing but a one-set can correspond ( $\$ 13)$. Hence it follows that $t=t^{\prime}+1$, and that all the one-sets which $t+1$ independent series satisfy make up a $t$-set. This is the $t$-set theorem.

24 . If the $t$ independent one-sets, or $t$-set, $\Sigma a_{q}^{p} E_{q}^{p}=0$, etc. and the $t^{\prime}$ independent one-sets, or $t^{\prime}$-set, $\Sigma a_{q}^{\prime p} E_{q}^{p}=0$, etc., are residual with respect to the one-set $E$, determined by the two series $h, k$, then the relations between the series $a, b, \ldots, a^{\prime}, b^{\prime}, \ldots$, and $h, k$ are as follows. No one of the series $a, b, \ldots$ is expressible by means of the rest and $h, k$, since the $t$ one-sets $\sum a_{q}^{p} E_{q}^{p}=0$, etc., are independent. If $h$ (or $k$ ) is dependent on $a, b, \ldots$, then, when $H$ ( or $K$ ) is expressed in the form $A P+B Q+\cdots$, the constant terms of the power series $P, Q, \cdots$ must all vanish. Similarly for the series $a^{\prime}, b^{\prime}, \ldots$, and $h, k$. The numbers $t, t^{\prime}$ can differ at most by unity.

(i) If $t^{\prime}=t-1$, the series $h, k$ are dependent on $a, b, \ldots$, and independent of $a^{\prime}, b^{\prime}, \ldots$. This is the case of $\S 23$ after omitting $a^{\prime}, b^{\prime}$.

If $t^{\prime}=t+1$, the series $h, k$ are dependent on $a^{\prime}, b^{\prime}, \ldots$, and independent of $a, b, \cdots$.

(ii) If $t^{\prime}=t$, the series $h, k$ can be so modified, without altering the whole one-set $E$ determined by them, that $h$ is dependent on $a, b, \ldots$ and independent of $a^{\prime}, b^{\prime}, \ldots$, and $k$ is dependent on $a^{\prime}, b^{\prime}, \ldots$ and independent of $a, b, \cdots$ This is not proved above.

Also if a $t$-set and $t^{\prime}$-set are residual with respect to a one-set $E$ they can be expressed as $t^{\prime}+1$ one-sets and $t+1$ one-sets respectively,

$$
\begin{aligned}
\Sigma a_{q}^{p} E_{q}^{p} & =0, \Sigma b_{q}^{p} E_{q}^{p}=0, \cdots \quad\left(t^{\prime}+1 \text { one-sets or } t \text {-set }\right), \\
\Sigma a_{q}^{p} E_{q}^{p} & =0, \Sigma b_{q}^{\prime p} E_{q}^{p}=0, \cdots \quad\left(t+1 \text { one-sets or } t^{\prime} \text {-set }\right),
\end{aligned}
$$

where the $t^{\prime}+1$ independent series $a, b, \cdots$ give the complete solution of the $t^{\prime}$-set, and the $t+1$ independent series $a^{\prime}, b^{\prime}, \ldots$ give the complete solution of the $t$-set. Here two of the $t^{\prime}+1$ one-sets are dependent on the rest, or two of the $t+1$ one-sets are dependent on the rest, or one one-set is dependent on the rest in each system; each of the series $h, k$ being among one or other of the $t+1$ and $t^{\prime}+1$ series.

St. PAUL's SChOOL, LONDON, Norember 5, 1903. 